\title{
An Electrocardiographic Early Repolarization Pattern in a Competitive Athlete: Is it a Sign of Danger?
}

\author{
Daniel B Petrov, MD, FESC ${ }^{*}$ (iD and Yoana D Petrova, M. Pharm ${ }^{2}$ (i) \\ ${ }^{1}$ Department of Emergency Cardiology, "Pirogov" Emergency Hospital, Bulgaria \\ ${ }^{2}$ School of Biological Sciences, Cardiff University, Sir Martin Evans Building, UK
}

*Corresponding author: Daniel B Petrov, MD, FESC, Department of Emergency Cardiology, "Pirogov" Emergency Hospital, 21 Totleben Ave, Sofia 1606, Bulgaria

\author{
Keywords \\ Early repolarization, Early repolarization pattern, \\ Electrocardiogram, Competitive athlete

\section{Abbreviations \\ ER: Early Repolarization; ERP: Early Repolarization Pattern; ECG: Electrocardiogram; SCD: Sudden Cardiac Death}

\section{Introduction}

The early repolarization (ER) is defined by a notch or slur in the J-point, which is often referred to as endQRS-notch or end-QRS-slur, while ST-segment elevation is not a required criterion. If the ST-segment is upward sloping and followed by an upright T-wave, the pattern should be described as ER with an ascending STsegment. If the ST-segment is horizontal or downward sloping, the pattern should be described as ER with a horizontal or descending ST-segment [1]. The J-point is the junction between the QRS complex and the STsegment and deviation of this point of the isoelectric line determines the presence of J deflection (J wave) $[2,3]$. $\mathrm{ER}$ is a common finding in young, healthy competitive athletes and appears to be a direct result of the electrical and structural remodeling or autonomic nervous system adaptation that occurs as a consequence of regular physical activity. It also depends on the athlete's ethnicity, age, gender, sporting discipline and level of training and competition [4].

The 12-leads electrocardiogram (ECG) can play a key role in the diagnosis of ERP, and many pre-participation screening protocols include an ECG, because is it a simple, inexpensive initial tool in the evaluation of both symptomatic and asymptomatic athletes. In the setting of pre-participation screening appropriate differential diagnosis between physiologic and pathologic STsegment elevation is of importance not only to prevent sport related sudden cardiac deaths (SCD), but also to avoid unnecessary disqualification from competition for changes that fall within the normal range for athletes [5].

This article describes the case of an asymptomatic competitive athlete who underwent ECG screening and was found to have early repolarization pattern (ERP) in context of a structurally normal heart. The authors discuss the ECG characteristics of ERP and proposed definition outlined in the 2015 consensus paper.

\section{Case Presentation}

A 21-year-old, white, professional marathon runner underwent cardiovascular assessment as a partof routine screening. He had no family history of cardiac disease or sudden cardiac deaths (SCD), and no abnormalities during a physical examination were found. Laboratory studies demonstrated normal levels of electrolytes, complete blood counts and negative cardiac necrosis markers. The transthoracic echocardiography was normal. A resting 12-leads electrocardiogram (ECG) demonstrated sinus rhythm with a rate of 62 beats per minute, a moderate concave ST-segment elevation 


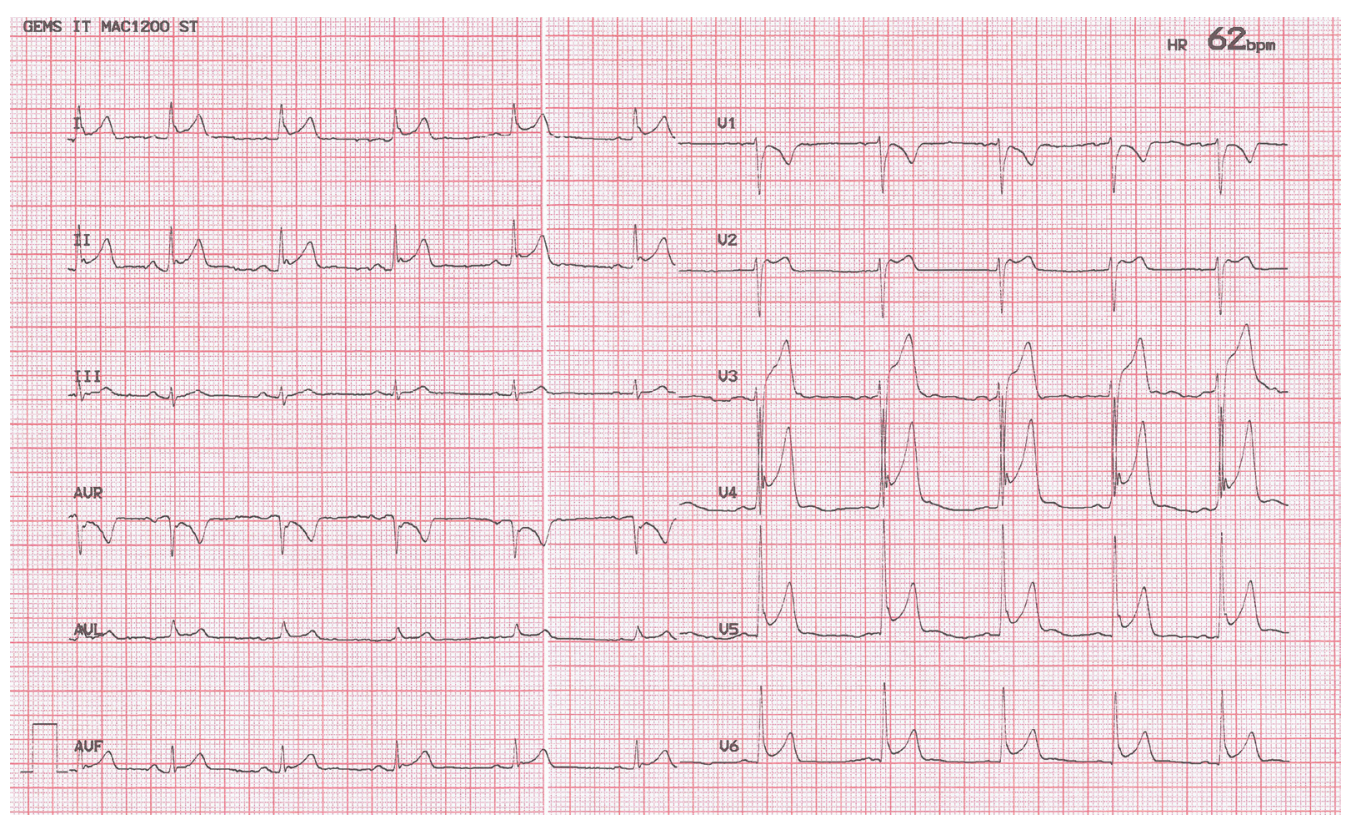

Figure 1: ECG showed moderate concave ST-segment elevation in leads I, II, aVL, aVF and more pronounced ST elevation in leads $V_{4}-V_{6}$, without reciprocal depression, followed by a positive $T$ waves.

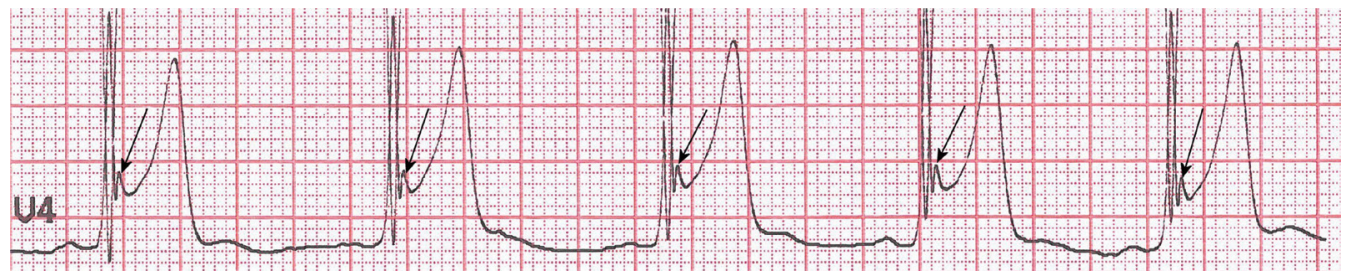

Figure 2: The strip demonstrated J-point elevation in lead $\mathrm{V}_{4}$ of $0.2 \mathrm{mV}$ and notch configuration, (arrows).

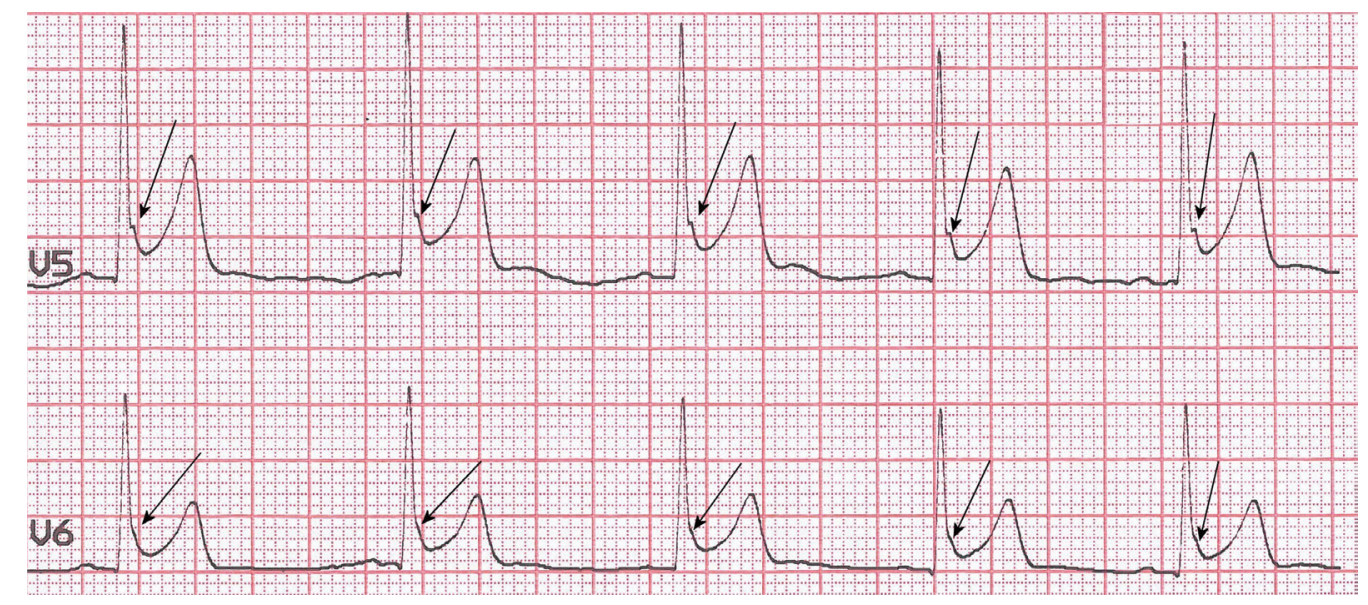

Figure 3: A magnified view of lead $\mathrm{V}_{5}-\mathrm{V}_{6}$ revealed J-point elevation with both notch and slur morphology in lead $\mathrm{V}_{5}$, progressing to only slur configuration in lead $V_{6}$ (arrows).

in leads I, II, aVL, aVF and more marked ST-elevation in leads $V_{4}$-to $V_{6}$ without reciprocal ST depression. The $T$ waves were prominent, slightly asymmetric and concordant with the QRS complexes (Figure 1). $A$ magnified view of lead $V_{4}$ clearly showed J-point elevation with a peak of $0.2 \mathrm{mV}$ and notch morphology (Figure 2 arrows), progressing to J-point elevation with a both notch and slur configuration in lead $V_{5}$ to only slur morphology in lead $\mathrm{V}_{6}$ (Figure 3 , arrows). This ECG finding strongly suggests an early repolarization pattern (ERP). Given the fact, that the subject was without any family history of SCD or any evidence of structural heart disease on the echocardiogram and benign ERP on ECG, he was allowed to continue with training and competition.

\section{Discussion}

The $J$ junction is the point at which the QRS complex 
ends and the ST-segment begins. The J point assessment depends on its deviation from the isoelectric line (elevated or depressed) which determines the presence of J-deflection (wave), and on its shape (notched or slurred). We have presented a classic example of ERP, with a notch at the J-point in $\mathrm{V}_{4}$ (Figure 2), progressing to J-point elevation with both a notch and slur morphology in $\mathrm{V}_{5}$ to only slur configuration in lead $\mathrm{V}_{6}$ (Figure 3 ). The elevated ST- segment is concave, the QRS complexes durations are narrow in the leads not containing notching or slur, and the $\mathrm{T}$ waves are relatively tall. Actually ERP is recognized in J-point notch or slur and this is often referred to as end QRS notch or end QRS slur. Consistent with the consensus report [1] an early repolarization (ER) is present if all of the following criteria are met: 1 ) There is an end QRS notch (J wave) or slur on the downslope of a prominent $\mathrm{R}$ wave, with or without ST-elevation; 2) J peak is $\geq 0.1 \mathrm{mV}$ in 2 or more contiguous leads of the 12-leads ECG, excluding leads $\mathrm{V}_{1}-\mathrm{V}_{3} ; 3$ ) QRS duration is $<120 \mathrm{~ms}$ (measured in leads in which a notch or slur is absent). Leads $V_{1}$ to $V_{3}$ have been excluded from the definition of ER to avoid confusion with the Brugada pattern.

Interest in ER has grown in recent years, because of the frequency with which that pattern is observed on routine ECG. ERP occurs in approximately $6 \%$ of the general population, and in $30 \%$ to $44 \%$ of athletes [6]. A retrospective cohort of 299 elite athletes of white ethnicity shows that ERP was present in one-third of the participants and this ECG pattern was not associated with fatal events after a mean follow-up of about 24 years [7]. The studies of Tikkanen, et al. [8] and Rosso, et al. [9] suggest that an upward-sloping ST-segment, followed by an upright T-wave in the presence of end-QRS notching or slurring is benign, whereas early repolarization with a horizontal or downward-sloping ST-segment is potentially more serious. In our case, ECG revealed upward-sloping ST-segment elevations and this is also compatible with the findings of Tikkanen, et al. [8] and Rosso, et al. [9] studies, since our subject did not have any history of aborted sudden death. More specifically, research has also suggested that vagal mediated bradycardia may increase $\mathrm{I}_{\mathrm{to}}$-mediated action potential current in the lateral left ventricle, leading to the presence of an end QRS notch or slur and ST-segment elevation in leads $\mathrm{V}_{4}$ to $\mathrm{V}_{6}$, which is suggested to have less arrhythmogenic potential [10]. With this in mind, most ECG interpretation guidelines for athletes state that based on current evidence all patterns of early repolarization without clinical markers of pathology should be considered benign variants in athletes. [11]. The impact of ethnicity is an important aspect upon cardiovascular adaptation in a competitive athlete. ER in black athletes may look different to the same condition in white ethnicity. In some young black men, the ST-segment is elevated in the mid precordial leads in combination with T-wave inversion as a normal variant. This entity may be the combination of ERP and a persistent juvenile T-wave pattern [11]. Recently, there has been recognition of a normal black athlete repolarization pattern which includes J-point elevation, convex ST-segment elevation, and T-wave inversion in leads $\mathrm{V}_{1}-\mathrm{V}_{4}$ occurring in $5-15 \%$ of black athletes. This pattern must be differentiated from T-wave inversions that are associated with hypertrophic cardiomyopathy or arrhythmogenic right ventricular cardiomyopathy [12]. Age also plays a role in this condition. Based on current evidence $T$-wave inversion in the anterior leads $\left(V_{1}-V_{3}\right)$ in adolescent athletes $<16$ years of age should not prompt further evaluation in the absence of symptoms, signs, or a family history of cardiac disease [13]. As discussed, ERP is a common finding among the athletic population and in the absence of relevant family history or symptoms, its clinical significance remains uncertain. While ERP is a common finding, its discovery on the 12lead ECG of an asymptomatic individual with no family history, does not currently warrant further assessment. There remain considerable challenges in determining which athletes with ERP are at increased risk of sudden cardiac death (SCD). There are two challenges facing the reduction of mortality, without increasing morbidity, for this group of subjects. First, athletes with ER have to be grouped according to the risk of developing SCD and be targeted accordingly. Second, it is necessary to identify which interventions exactly can achieve such goal [14].

\section{Conclusion}

The presence of J peak $>0.1 \mathrm{mV}$, with notching or/ and slurring configuration, excluding leads $\mathrm{V}_{1}-\mathrm{V}_{3}$ is the cornerstone for diagnosis of ERP, while the ST-segment elevation is not a required criterion. ERP is associated with a significant clinical attention and the appropriate interpretation of the ECG in young, competitive athletes is challenging. For the specialists and clinicians it is very important to observe and identify the ERP and to make initial risk stratification based on history and ECG findings.

\section{Conflicts of Interest}

The two authors have no conflicts of interest to disclose.

\section{Funding}

None.

\section{References}

1. Macfarlane PW, Antzelevitch $C$, Haissaguerre M, Huikuri HV, Potse M, et al. (2015) The early repolarization pattern: A Consensus paper. J Am Coll Cardiol 66: 470-477.

2. Antzelevitch C, Yan GX, Ackerman MJ, Borggrefe $M$, Corrado D, et al. (2016) J-wave syndromes expert consensus conference report: Emerging concepts and gaps in knowledge. Heart Rhythm 13: e295-e324.

3. Antzelevitch C, Yan GX (2010) J wave syndromes. Heart Rhythm 7: 549-558.

4. Sharma S (2003) Athlete's heart--effect of age, sex, 
ethnicity and sporting discipline. Exp Physiol 88: 665-669.

5. Corrado D, Mc Kenna WJ (2007) Appropriate interpretation of the athlete's electrocardiogram saves lives as well as money. Eur Heart J 28: 1920-1922.

6. Al-Zaiti SS, Hoster D, Kozik TM, Pelter MM, Carey MG, et al. (2014) Repolarization abnormalities in young athletes. Am J Crit Care 23: 345-346.

7. Serra-Grima R, Donate M, Alvarez-Garcia J, BarradasPires A, Ferrero A, et al. (2015) Long-term follow-up of early repolarization in elite athletes. Am J Med 128: 192. e1-192.e9.

8. Tikkanen JT, Junttila MJ, Anttonen O, Aro AL, Luttinen $S$, et al. (2011) Early repolarization: Electrocardiographic phenotypes associated with favorable long-term outcome. Circulation 123: 2666-2673.

9. Rosso R, Glikson E, Belhassen B, Halkin A, Steinvil A et al. (2012) Distinguishing benign from malignant early repolarization: The value of the ST-segment morphology. Heart Rhythm 9: 225-229.

10. Hlaing T, DiMino T, Kowey PR, Yan GX (2005) ECG repolarization waves: Their genesis and clinical implications. Ann Noninvasive Electrocardiol 10: 211-223.

11. Sharma S, Drezner JA, Baggish A, Papadakis M, Wilson MG, et al. (2018) International recommendations for electrocardiographic interpretation in athletes. Eur Heart $\mathrm{J}$ 39: 1466-1480.

12. Wang K, Asinger RW, Marriot HJL (2003) ST-segment elevation in conditions other than acute myocardial infarction. N Engl J Med 349: 2128-2135.

13. Hermelin MJ, Prutkin MJ (2018) Black athlete electrocardiographic repolarization pattern. J Electrocardiol 51: $680-682$

14. Aagaard P, Baranowski B, Aziz P, Phelan D (2016) Early repolarization in athletes: A Review. Circ Arrhythm Electrophysiol 9: e003577. 